\title{
Levetiracetam for Benzodiazepine-Refractory Status Epilepticus
}

\author{
Priyanka Madaan ${ }^{1} \cdot$ Jitendra Kumar Sahu ${ }^{1}$
}

Received: 3 May 2020 / Accepted: 5 May 2020 / Published online: 15 May 2020

(C) Dr. K C Chaudhuri Foundation 2020

With the advent of newer antiseizure medications (ASM) with a better safety profile, these newer drugs are superseding the older ones in the management of epilepsy. However, the overall outcomes in newly-diagnosed epilepsy remain unchanged [1]. Despite the ingress of newer ASM, the management guidelines for convulsive status epilepticus (SE) have been the same [2]. The most important reason for this is the lack of high-quality supporting evidence for newer ASM in SE, which highlights the difficulties in conducting large randomized controlled trials (RCT) for emergent conditions like SE.

Convulsive $\mathrm{SE}$ is the commonest neurological emergency encountered by pediatricians and pediatric neurologists. Benzodiazepines are the first-line treatment choice. However, when benzodiazepine-refractory status epilepticus (BRSE) evolves, guidelines recommend intravenous phenytoin or fosphenytoin as the preferred drug options [2]. However, the evidence-base for such recommendation is weak $[2,3]$.

Considering the better safety profile and feasibility of rapid administration of levetiracetam, two recent large RCTs with a superiority design compared the efficacy and safety of levetiracetam $(40 \mathrm{mg} / \mathrm{kg})$ with phenytoin $(20 \mathrm{mg} / \mathrm{kg})$ as the initial choice for BRSE in children $[4,5]$. However, both these trials did not report any significant difference between phenytoin and levetiracetam for any outcome.

With fosphenytoin substituting phenytoin for BRSE in many countries, a recent open-label RCT from India by Nalisetty and colleagues compared levetiracetam with fosphenytoin for the same indication in children [6]. The study reported seizure cessation to the tune of $69 \%$ and $94 \%$ in

Jitendra Kumar Sahu

jsh2003@gmail.com

Priyanka Madaan

doc.priyanka72@gmail.com

1 Pediatric Neurology Unit, Department of Pediatrics, Postgraduate Institute of Medical Education and Research (PGIMER), Chandigarh, India fosphenytoin $(n=29)$ and levetiracetam $(n=32)$ group, respectively. Strikingly, 7 out of 9 children who did not respond to fosphenytoin, responded to levetiracetam. Although the study had a small sample size (61 patients) and was not powered enough to conclude assertively, this was an applaudable effort considering the challenges and problems in conducting research in SE.

Levetiracetam may be an effective alternative drug to phenytoin or fosphenytoin for BRSE in children [4-6]. Also, the sequential administration of these two drugs (in any sequence) may further reduce the failure rate $[5,6]$. This is an era of precision medicine, and levetiracetam may be advantageous when febrile status epilepticus is related to SCN1A genetic variants where fosphenytoin may be less effective. Future research aiming at identification of the best therapeutic protocol for SE with minimum failure rate, shorter time to cessation, and reduced progression to refractory SE is the need of the hour. Furthermore, future research should also assess the choice of ASM based on the underlying etiology.

\section{Compliance with Ethical Standards}

Conflict of Interest None.

\section{References}

1. Chen Z, Brodie MJ, Liew D, Kwan P. Treatment outcomes in patients with newly diagnosed epilepsy and new antiepileptic drugs: a 30-year longitudinal cohort study. JAMA Neurol. 2018;75:279-86.

2. Glauser T, Shinnar S, Gloss D, et al. Evidence based guidelines: treatment of convulsive status epilepticus in children and adults: report of the guideline committee of the American Epilepsy Society. Epilepsy Curr. 2016;16:48-61.

3. McTague A, Martland T, Appleton R. Drug management for acute tonic clonic convulsion including convulsive status epilepticus in children. Cochrane Database Syst Rev. 2018;1:CD001905.

4. Lyttle MD, Rainford NEA, Gamble C, et al. Levetiracetam versus phenytoin for second-line treatment of paediatric convulsive status epilepticus (EcLiPSE): a multicenter, open-label, randomized trial. Lancet. 2019;393:2125-34. 
5. Dalziel SR, Borland ML, Furyk J, et al. Levetiracetam versus phenytoin for second-line treatment of convulsive status epilepticus in children (ConSEPT): an open- label, multicentre, randomised controlled trial. Lancet. 2019;393:2135-45.

6. Nalisetty S, Kandasamy S, Sridharan B, Vijayakumar V, Sangaralingam T, Krishnamoorthi N. Clinical effectiveness of levetiracetam compared to fosphenytoin in the treatment of benzodiazepine refractory convulsive status epilepticus. Indian J Pediatr. 2020. https://doi.org/10.1007/s12098-020-03221-2.

Publisher's Note Springer Nature remains neutral with regard to jurisdictional claims in published maps and institutional affiliations. 